\title{
A LATTICE CONTROL MODEL OF FUZZY DYNAMICAL SYSTEMS IN STATE-SPACE *
}

\author{
PETROS MARAGOS, GIORGOS STAMOU and \\ SPYROS TZAFESTAS \\ National Technical University of Athens, \\ Dept. of Electrical 83 Computer Engineering, \\ Zografou, 15773 Athens, Greece.
}

\begin{abstract}
Lattice control can unify nonlinear control systems where the basic vector and signal superpositions or transformations are based on the lattice supremum and infimum. In this paper we introduce a special case of lattice control that can model fuzzy dynamical systems in state space. Vector and signal transformations are represented as lattice dilations or erosions. The state and output responses are computed via supremal convolutions based on fuzzy norms. Causality and stability issues are studied. Finally, solutions to the controllability and observability problem are found using lattice adjunctions.
\end{abstract}

Key words: Nonlinear control, lattice morphology, fuzzy systems, minimax algebra.

\section{Lattice Model for Max and Min Control}

In $[7,8,6]$ a unified model was proposed based on lattice theory for large classes of nonlinear control systems, such as discrete event dynamical systems, recursive morphological filters, and fuzzy dynamical systems. Lattice morphology $[9,3]$ is ideally suited to studying such systems because all vector and signal operations and mappings involved can be expressed as morphological operators, and solutions to important control issues such as responses, stability and controllability are obtained using simple lattice-theoretic morphological concepts. In this paper we examine a special case of lattice control systems applicable to fuzzy dynamical systems.

In classical linear control the state vectors, the input/output signals, and the system matrices take values from the field of reals equipped with standard addition and multiplication. In lattice control we take the set $\mathbb{V}$ of scalars to be a complete sublattice of $\overline{\mathbb{R}}$ and equip it with the standard real number ordering $\leq$ and four binary operations:

(A). A generalized 'addition', which will be the supremum $\vee$ on reals.

$\left(\mathrm{A}^{\prime}\right)$. A 'dual addition', which will be the infimum $\wedge$ on reals.

(M). A commutative generalized 'multiplication' $\star$ under which: (i) $\mathbb{V}$ is a monoid (i.e., semigroup possessing an identity) with identity $V_{\text {id }}$ and null element $V_{\text {inf }}=\bigwedge \mathbb{V}$, and (ii) $\star$ is a scalar dilation, i.e., distributes over any

* This paper was published in: Mathematical Morphology and Its Application to Image and Signal Processing, Edited by J. Goutsias, L. Vincent and D. Bloomberg, Kluwer Academic Publishers, Boston, 2000, pp.61-70 (Proc. ISMM 2000). This research was supported by the Greek Secretariat for Research and Technology under Grants EПET - 98ГT26 and $\Pi E N E \Delta-99 E \Delta 164$. 
supremum.

$\left(\mathrm{M}^{\prime}\right)$. A commutative 'dual multiplication' $\star^{\prime}$ under which: (i) $\mathbb{V}$ is a monoid with identity $V_{\mathrm{id}}{ }^{\prime}$ and null element $V_{\text {sup }}=\bigvee \mathbb{V}$, and (ii) $\star^{\prime}$ is a scalar erosion, i.e., distributes over any infimum.

We group the above requirements into three assumptions: (LCA1). $(\mathbb{V}, \vee, \wedge)$ is a complete infinitely-distributive lattice. (LCA2). ( $\mathbb{V}, \star)$ is a commutative monoid, and $\star$ is a dilation. (LCA3). ( $\left.\mathbb{V}, \star^{\prime}\right)$ is a commutative monoid, and $\star^{\prime}$ is an erosion.

Under the above assumptions $\left(\mathbb{V}, \vee, \wedge, \star, \star^{\prime}\right)$ becomes a commutative complete lattice-ordered double monoid (CLODUM). This will be the most general and minimally required algebraic structure we consider for the set of scalars. In our model, all the vectors/matrices/signals take values from $\mathbb{V}$, and their 'addition' is done via pointwise sup or inf. The most important abstraction is 'multiplication' of two matrices. ${ }^{1}$ Thus, the generalized max-^ 'product' of a matrix $\mathbf{Q}=\left[q_{i j}\right] \in \mathbb{V}^{m \times \ell}$ with a matrix $\mathbf{R}=\left[r_{i j}\right] \in \mathbb{V}^{\ell \times n}$ yields a matrix $\mathbf{P}=\left[p_{i j}\right] \in \mathbb{V}^{m \times n}$ defined by:

$$
\mathbf{P}=\mathbf{Q} \text { 囚 } \mathbf{R}, \quad p_{i j}=\bigvee_{k=1}^{\ell} q_{i k} \star r_{k j}
$$

The state equations of the max control model are:

$$
\begin{aligned}
& \mathbf{x}(k+1)=\mathbf{A} \text { 刃 } \mathbf{x}(k) \vee \mathbf{B} \text { 囚 } \mathbf{u}(k) \\
& \mathbf{y}(k)=\mathbf{C} \otimes \mathbf{x}(k) \vee \mathbf{D} \otimes \mathbf{u}(k)
\end{aligned}
$$

where $k$ is a discrete time index. We assume a $n$-dimensional state vector $\mathbf{x}=\left[x_{1}, x_{2}, \ldots, x_{n}\right]^{t} \in \mathbb{V}^{n}$, a $p$-dimensional input $\mathbf{u} \in \mathbb{V}^{p}$, and an $r$-dimensional output $\mathbf{y} \in \mathbb{V}^{r}$. Therefore, the four matrices have the following sizes: $\mathbf{A} \in$ $\mathbb{V}^{n \times n}, \mathbf{B} \in \mathbb{V}^{n \times p}, \mathbf{C} \in \mathbb{V}^{r \times n}$, and $\mathbf{D} \in \mathbb{V}^{r \times p}$. By replacing $\vee$ with $\wedge$ and 因 with a dual matrix 'product' $⿴ 囗$ ', where a row and a column vector are 'multiplied' via a min- $\star$ ' operation, we obtain a dual model that describes the state-space dynamics of min control systems.

By specifying the scalar 'multiplication' $\star$ and its dual $\star^{\prime}$, we obtain a large variety of classes of nonlinear dynamical systems that are described by the above unified lattice control model. Two such choices are:

(1) Max-Sum Control where $\mathbb{V}=\overline{\mathbb{R}}$ and $\star=+$. Such systems (with special choices of $\mathbf{A}, \mathbf{B}, \mathbf{C}, \mathbf{D})$ have been used in $[1,4,8]$ to model the dynamics of certain classes of discrete event dynamical systems (DEDS) as applied to material flow in manufacturing systems and related scheduling problems. The underlying nonlinear matrix operations are the basis of the minimax algebra [2], which has found numerous applications in DEDS and operations research. In typical applications of DEDS, the states $x_{i}(k)$ may represent the start-up or completion time of the $k$-th cycle of machine $i$, the input $\mathbf{u}$ represents availability times of parts, $\mathbf{y}$ represents exit times, and the elements of the matrices A, B, C, D represent service/delay times or activity durations. Further, the

1 Notation: If $\mathbf{M}=\left[m_{i j}\right]$ is a matrix, its $(i, j)$ th element is also denoted as $\{\mathbf{M}\}_{i j}=m_{i j}$. Similarly, if $\mathbf{x}=\left[x_{i}\right]$ is a vector, its $i^{\text {th }}$ element is denoted as $\{\mathbf{x}\}_{i}$ or simply $x_{i}$. 
max-sum control model can also capture the dynamics of recursive morphological filters described by max-sum difference equations [8].

(2) Max-Tnorm Control: There are many types of nonlinear control systems where the elements of the state, input and output vectors represent fuzzy set memberships [12], possibilities, or probabilities. Examples include fuzzy control systems, probabilistic automata, fuzzy classifiers, as well as certain types of neural nets with nonlinear combinations of inputs; surveys of such fuzzy systems can be found in [5]. Fuzzy state-space models can be useful for qualitative modeling of problems with large number of states where quantitative modeling is impossible. The dynamics of large classes of such systems can be described via the lattice control model by restricting the set of scalars to be $\mathbb{V}=[0,1]$ and using a fuzzy intersection norm (a.k.a. 'triangular-norm') $T(a, b) \triangleq a \star b$ as the scalar 'multiplication'. This paper deals with this special case of lattice control.

\section{Max-Tnorm Control}

A fuzzy intersection norm, in short a Tnorm, is a binary operation $T$ : $[0,1] \rightarrow[0,1]$ that satisfies the following conditions [5]: For all $a, b, c \in[0,1]$

F1. $T(a, 1)=a$ and $T(a, 0)=0$ (boundary conditions).

F2. $T(a, T(b, c))=T(T(a, b), c)$ (associativity).

F3. $T(a, b)=T(b, a)$ (commutativity).

F4. $b \leq c \Longrightarrow T(a, b) \leq T(a, c)$ (increasing).

For the Tnorm to satisfy the general algebraic conditions we require from the set of scalars, it must also satisfy the following:

F5. $T$ is a continuous function.

Conditions F1-F3 make $([0,1], T)$ a commutative monoid with identity $V_{\text {id }}=1$ and null $V_{\text {inf }}=0$. Conditions F4-F5 suffice to make $T$ a scalar dilation with respect to any argument, as proven next.

Proposition 1 . Let $T$ be a continuous fuzzy intersection norm. Then, the operator $x \mapsto T(x, a)$, for any arbitrary fixed $a \in[0,1]$, is a dilation.

Proof: Consider a (finite or infinite) collection $\left\{x_{j}: j \in J\right\}$ of points in $[0,1]$ with $x=\bigvee_{j \in J} x_{j}$. Since $[0,1]$ is compact, we can find an increasing subsequence $\left\{x_{k}=x_{j_{k}}\right\}$, such that $x_{k} \leq x_{k+1}$ and $x=\bigvee_{k} x_{k}=\lim _{k \rightarrow \infty} x_{k}$. Since $T$ is increasing, $T\left(x_{k}, a\right)$ is also an increasing sequence that converges to its supremum $\bigvee_{k} T\left(x_{k}, a\right)$. Further, $\bigvee_{k} T\left(x_{k}, a\right) \leq \bigvee_{j} T\left(x_{j}, a\right) \leq T(x, a)$. Finally, since $T$ is continuous, we have $\bigvee_{k} T\left(x_{k}, a\right)=\lim _{k \rightarrow \infty} T\left(x_{k}, a\right)=T(x, a)$. This yields

$$
T\left(\bigvee_{j} x_{j}, a\right)=\bigvee_{j} T\left(x_{j}, a\right)
$$

which proves that $T$ is a scalar dilation.

As a 'dual multiplication' we may use a fuzzy union norm $T^{\prime}(a, b) \triangleq a \star^{\prime} b$, where $T^{\prime}$ satisfies F2-F5 and a dual boundary condition: $\mathrm{F} 1^{\prime} . T^{\prime}(a, 0)=a$ and $T^{\prime}(a, 1)=1$ (dual boundary conditions). 
Clearly, $\left([0,1], T^{\prime}\right)$ is a commutative monoid, and $T^{\prime}$ is an erosion. Choosing in the lattice control model the above set of scalars and 'multiplications' among them creates the case of $\max -T$ and $\min -T^{\prime}$ control systems, obtained by replacing in the general state equations of lattice control the general matrix 'product' $⿴$ and its dual $\boldsymbol{\otimes}^{\prime}$ with the following max- $T$ and $\min -T^{\prime}$ versions:

$$
\begin{aligned}
& \mathbf{P}=\mathbf{Q} \square_{T} \mathbf{R}, p_{i j}=\bigvee_{k} T\left(q_{i k}, r_{k j}\right) \\
& \mathbf{P}=\mathbf{Q} \square_{T}^{\prime} \mathbf{R}, p_{i j}=\bigwedge_{k} T^{\prime}\left(q_{i k}, r_{k j}\right)
\end{aligned}
$$

The most obvious choice for the $T$ norm and its dual norm $T^{\prime}$ are the min and max, respectively. But there are also numerous other choices [5].

\section{Vector and Signal Lattice Operators}

The space of vectors and the space of signals with values from the lattice $\mathbb{V}$ are special cases of function lattices. The underlying set of these lattices is the set $\mathcal{L}=\mathbb{V}^{E}$ of all functions mapping an arbitrary nonempty set $E$ into $\mathbb{V}$. In particular, if $E=\{1,2, \ldots, n\}$, then $\mathcal{L}$ becomes the set of all $n$-dimensional vectors ( $n$-tuples) $\left[x_{1}, \ldots, x_{n}\right]^{t}$ with elements from $\mathbb{V}$. If $E=\mathbb{Z}$, then $\mathcal{L}$ becomes the set of all discrete-time signals with values from $\mathbb{V}$. The set $\mathcal{L}$ becomes a complete infinitely distributive lattice if we define on it the standard pointwise partial ordering $\leq$, supremum $\vee$, and infimum $\wedge$ induced by $\mathbb{V}$.

Pointwise 'multiplication' of a lattice element $F \in \mathcal{L}=\mathbb{V}^{E}$ by a scalar $a \in \mathbb{V}$ yields elementary dilations on $\mathcal{L}$ that are called translations $\tau_{a}(F)(x)$ $\triangleq T(F(x), a), x \in E$. An operator $\psi$ on $\mathcal{L}$ is called translation invariant iff it commutes with any translation, i.e., $\psi \tau=\tau \psi$ for all $\tau$. All the above concepts apply as well for the dual translations $\tau_{a}^{\prime}(F)(x) \triangleq T^{\prime}(F(x), a)$, which are elementary erosions on $\mathcal{L}$.

More general dilations and erosions on the function lattice $\mathcal{L}=\mathbb{V}^{E}$ can be decomposed into suprema and infima of scalar dilations and erosions on $\mathbb{V}$, respectively.

Proposition 2 ([3]). Let $\mathbb{V}$ be a complete lattice and $E$ an arbitrary nonempty set. The pair $(\varepsilon, \delta)$ is an adjunction on the function lattice $\mathbb{V}^{E}$ iff for every $x, y \in E$ there exists an adjunction $\left(\varepsilon_{x, y}, \delta_{x, y}\right)$ on $\mathbb{V}$ such that

$$
\delta(F)(y)=\bigvee_{x \in E} \delta_{x, y}(F(x)) \quad, \quad \varepsilon(G)(x)=\bigwedge_{y \in E} \varepsilon_{y, x}(G(y))
$$

If we define the impulse functions $q$ and their duals $q^{\prime}$

$$
q_{z, v}(x)=\left\{\begin{array}{l}
v, x=z \\
0, x \neq z
\end{array} \quad, \quad q_{z, v}^{\prime}(x)=\left\{\begin{array}{l}
v, x=z \\
1, x \neq z
\end{array}\right.\right.
$$

we can enable the decomposition (5) by defining the scalar dilations to be

$$
\delta_{x, y}(v)=\delta\left(q_{x, v}\right)(y), \quad v \in \mathbb{V}
$$

and $\varepsilon_{y, x}$ to be the adjoint erosion of $\delta_{x, y}$. 


\subsection{Vector Lattice}

Consider now the vector space $\mathcal{L}=\mathbb{V}^{n}$, equipped with the partial ordering $\mathbf{x} \leq \mathbf{y}$, which means $x_{i} \leq y_{i} \forall i$, the supremum $\mathbf{x} \vee \mathbf{y}=\left[x_{i} \vee y_{i}\right]$ and the infimum $\mathbf{x} \wedge \mathbf{y}=\left[x_{i} \wedge y_{i}\right]$ between any vectors $\mathbf{x}, \mathbf{y} \in \mathcal{L}$. Then, $(\mathcal{L}, \vee, \wedge)$ is a complete infinitely distributive lattice. Elementary vector dilations are the vector translations $\tau_{a}(\mathbf{x})=\left[T\left(x_{i}, a\right)\right]$ and their duals $\tau_{a}^{\prime}(\mathbf{x})=\left[T^{\prime}\left(x_{i}, a\right)\right]$. By defining as 'impulse functions' the unit vectors $\mathbf{e}$ and their duals $\mathbf{e}^{\prime}$

$$
\mathbf{e}_{i} \triangleq[0, \ldots, 0,1,0, \ldots, 0]^{t}, \quad \mathbf{e}_{i}^{\prime} \triangleq[1, \ldots, 1,0,1, \ldots, 1]^{t}
$$

each vector $\mathbf{x}=\left[x_{1}, \ldots, x_{n}\right]^{t}$ can be represented as a max of translated impulse vectors or as a min of dual-translated dual impulse vectors

$$
\mathbf{x}=\bigvee_{i=1}^{n} \tau_{x_{i}}\left(\mathbf{e}_{i}\right)=\bigwedge_{i=1}^{n} \tau_{x_{i}}^{\prime}\left(\mathbf{e}_{i}^{\prime}\right)
$$

More general forms of vector dilation $\left(\delta_{M}\right)$ and erosion $\left(\varepsilon_{M}\right)$ are, respectively, the max- $T$ and min- $T^{\prime}$ 'product' of a matrix $\mathbf{M}$ with an input vector:

$$
\delta_{M}(\mathbf{x}) \triangleq \mathbf{M} \square_{T} \mathbf{x}, \quad \varepsilon_{M}(\mathbf{x}) \triangleq \mathbf{M} \square_{T}^{\prime} \mathbf{x}
$$

A vector operator $\psi$ on $\mathcal{L}$ is (dual-)translation invariant iff it commutes with any vector (dual-)translation.

Theorem 1 ([6]). (a) Any translation invariant dilation $\delta$ on the vector lattice $\mathcal{L}=[0,1]^{n}$ can be represented as a matrix-based dilation $\delta_{M}$ where $\mathbf{M}=\left[m_{i j}\right]$ with $m_{i j}=\left\{\delta\left(\mathbf{e}_{j}\right)\right\}_{i}$, and vice-versa.

(b) Any dual-translation invariant erosion $\varepsilon$ on $\mathcal{L}$ can be represented as a matrix-based erosion $\varepsilon_{M^{\prime}}$ where $\mathbf{M}^{\prime}=\left[m_{i j}^{\prime}\right]$ with $m_{i j}^{\prime}=\left\{\varepsilon\left(\mathbf{e}_{j}^{\prime}\right)\right\}_{i}$, and viceversa.

Given a vector dilation $\delta(\mathbf{y})=\mathbf{M} \square_{T} \mathbf{y}$ with $\mathbf{M}=\left[M_{i j}\right]$, what is its adjoint erosion $\varepsilon$ ? The scalar adjoint erosion stems from a binary operation $\xi$ : $[0,1]^{2} \rightarrow[0,1]$ defined by

$$
\xi(w, a) \triangleq \sup \{v: T(v, a) \leq w\}
$$

For example, the adjoints of the minimum and product Tnorms are:

$$
\begin{gathered}
T(v, a)=\min (v, a) \Longrightarrow \xi(w, a)=\frac{1+w}{2}+\left(\frac{1-w}{2}\right) \operatorname{sign}(w-a) \\
T(v, a)=a v \Longrightarrow \xi(w, a)=\min \left(\frac{w}{a}, 1\right)
\end{gathered}
$$

where $\operatorname{sign}(r)=1$ if $r \geq 0$ and -1 else. If we consider the scalar dilations $\delta_{i j}(v)=\left\{\delta\left(\mathbf{e}_{i, v}\right)\right\}_{j}=T\left(v, m_{j i}\right)$ then their adjoint scalar erosions are $\varepsilon_{j i}(w)=$ $\xi\left(w, m_{j i}\right)$. Thus, according to the decomposition (5), the adjoint vector erosion is

$$
\varepsilon(\mathbf{x})=\mathbf{M}^{t} \square_{\xi} \mathbf{x}, \quad\left\{\mathbf{M}^{t} \square_{\xi} \mathbf{x}\right\}_{i} \triangleq \bigwedge_{j} \xi\left(x_{j}, m_{j i}\right)
$$

where $(\cdot)^{t}$ denotes matrix transposition. 


\subsection{Signal Lattice}

Consider the set $\mathbb{V}^{\mathbb{Z}}$ of all discrete-time signals $f: \mathbb{Z} \rightarrow \mathbb{V}$ with values form $\mathbb{V}$. Equipped with pointwise sup $\vee$ and inf $\wedge$, this becomes a complete infinitely distributive lattice $\mathcal{L}$ with partial order the pointwise signal relation $\leq$. The signal translations are the operators $\tau_{i, v}(f)(k)=T(f(k-i), v)$, where $(i, v) \in$ $\mathbb{Z} \times \mathbb{R}$ and $f(k)$ is an arbitrary input signal. A signal operator on $\mathcal{L}$ is called translation invariant iff it commutes with any such translation. Consider now two elementary signals, called the impulse $q$ and the dual impulse $q^{\prime}$ :

$$
q(k) \triangleq\left\{\begin{array}{ll}
1, & k=0 \\
0, & k \neq 0
\end{array}, \quad q^{\prime}(k) \triangleq \begin{cases}0, & k=0 \\
1, & k \neq 0\end{cases}\right.
$$

Then every signal $f$ can be represented as a sup of translated impulses or as inf of dual-translated dual impulses:

$$
f(k)=\bigvee_{i} T[f(i), q(k-i)]=\bigwedge_{i} T^{\prime}\left[f(i), q^{\prime}(k-i)\right]
$$

General signal dilation and erosion can result, respectively, from the sup- $T$ convolution $\bigcirc_{T}$ and the inf- $T^{\prime}$ convolution $\bigcirc_{T}^{\prime}$ of two signals $f$ and $g$ defined by

$$
f \bigcirc_{T} g(k) \triangleq \bigvee_{i} T[f(i), g(k-i)], \quad f \bigcirc_{T}^{\prime} g(k) \triangleq \bigwedge_{i} T^{\prime}[f(i), g(k-i)]
$$

The following theorem characterizes all translation invariant signal dilation or erosion systems as nonlinear convolutions of the above type.

Theorem 2 ([6]). (a) An operator $\Delta$ on the signal lattice $[0,1]^{\mathbb{Z}}$ is a translation invariant dilation iff it can be represented as the sup- $T$ convolution of the input signal with the system's impulse response $h=\Delta(q)$.

(b) An operator $E$ on the signal lattice $[0,1]^{\mathbb{Z}}$ is a dual-translation invariant erosion iff it can be represented as the inf- $T^{\prime}$ convolution of the input signal with the system's dual impulse response $h^{\prime}=E\left(q^{\prime}\right)$.

Given a signal dilation $\Delta(f)=f \bigcirc_{T} h$ and its representation via scalar dilations as

$$
\Delta(f)(\ell)=\bigvee_{k \in \mathbb{Z}} \Delta_{k, \ell}(f(k)), \quad \Delta_{k, \ell}(v)=T[v, h(\ell-k)]
$$

it follows from the decomposition (5) that its adjoint signal erosion is

$$
E(g)(k)=\bigwedge_{\ell \in \mathbb{Z}} \xi[g(\ell), h(\ell-k)]
$$




\section{State and Output Responses}

The basic state-space model of a max- $T$ control system can now be represented via matrix-based dilations:

$$
\begin{array}{rlll}
\mathbf{x}(k+1) & =\delta_{A}[\mathbf{x}(k)] & \vee & \delta_{B}[\mathbf{u}(k)] \\
\mathbf{y}(k) & =\delta_{C}[\mathbf{x}(k)] & \vee & \delta_{D}[\mathbf{u}(k)]
\end{array}
$$

Solving the state equations by using induction on $k$ yields the state response:

$$
\begin{aligned}
\mathbf{x}(k) & =\mathbf{A}^{(k)} \square_{T} \mathbf{x}(0) \vee\left(\bigvee_{i=0}^{k-1} \mathbf{A}^{(k-1-i)} \square_{T} \mathbf{B} \square_{T} \mathbf{u}(i)\right) \\
& =\delta_{A}^{k}[\mathbf{x}(0)] \vee\left(\bigvee_{i=0}^{k-1} \delta_{A}^{k-1-i} \delta_{B}[\mathbf{u}(i)]\right)
\end{aligned}
$$

where $\mathbf{A}^{(k)}$ denotes the $k$-fold max- $T$ matrix 'product' of $\mathbf{A}$ with itself for $k \geq 1$ and $\mathbf{A}^{(0)}=\mathbf{I}_{n}$ where $\mathbf{I}_{n}$ is the $n \times n$ identity matrix.

The above result yields in turn the output response:

$$
\mathbf{y}(k)=\underbrace{\delta_{C} \delta_{A}^{k}[\mathbf{x}(0)]}_{\text {'zero'-input resp. }} \vee \underbrace{\left(\bigvee_{i=0}^{k-1} \delta_{C} \delta_{A}^{k-1-i} \delta_{B}[\mathbf{u}(i)]\right) \vee \delta_{D}[\mathbf{u}(k)]}_{\mathbf{y}_{z s}(k) \triangleq \text { 'zero'-state resp. }}
$$

Thus, the output response is found to consist of two parts: (i) the 'zero'-input response which is due only to the initial conditions $\mathbf{x}(0)$ and assumes an input equal to 0 , and (ii) the 'zero'-state response which is due only to the input $\mathbf{x}(0)$ and assumes initial conditions equal to 0.

For single-input single-output systems the mapping $u(k) \mapsto y_{z s}(k)$ can be viewed as a translation invariant dilation system $\Delta$. Hence, the 'zero'-state response can be found as the sup- $T$ convolution of the input with the system's impulse response $h=\Delta(q)$ :

$$
y_{z s}(k)=\Delta(u)(k)=\bigvee_{\ell} T[u(\ell), h(k-\ell)]
$$

Assuming the system is initially at rest, its impulse response is found to be

$$
h(k)=\left\{\begin{array}{lr}
0, & k<0 \\
\mathbf{D}, & k=0 \\
\mathbf{C} \square_{T} \mathbf{A}^{(k-1)} \square_{T} \mathbf{B}, & k>0
\end{array}\right.
$$

The last two results can be easily extended to multi-input multi-output systems.

\section{Causality, Stability}

A max- $T$ control system initially at rest can be viewed as a translation invariant dilation system $\Delta$ mapping the input $u$ to the output $y$. (Assume for brevity single-input single-output systems.) Let $h=\Delta(q)$ be the impulse response of 
$\Delta$. A useful bound for signals $f(k)$ processed by such systems is their global supremum

$$
\|f\|_{\vee} \triangleq \bigvee_{k \in \mathbb{Z}} f(k)
$$

which can be viewed as a semi-norm. Such systems are called bounded-input bounded-output (BIBO) stable iff a bounded input yields a bounded output, i.e., if $\|u\|_{\vee}<1 \Longrightarrow\|y\|_{\vee}<1$. The following theorem provides us with simple algebraic criteria for checking the causality and stability of max- $T$ control systems based on their impulse response.

Theorem 3 ([6]). Consider a max-Tnorm control system $\Delta$ initially at rest and let $h=\Delta(q)$ be its impulse response. (a) The system is causal iff $h(k)=0$ for all $k<0$. (b) The system is BIBO stable iff $\|h\|_{\vee}<1$.

\section{Controllability, Observability}

A max- $T$ control system is controllable if the following system of nonlinear equations can be solved and provide the vector $\mathbf{u}=[u(0), u(1), \ldots, u(N-1)]^{t}$ of input values required to drive the system from the initial state $\mathbf{x}(0)$ to any desired state $\mathbf{x}(N)$ in $N$ steps:

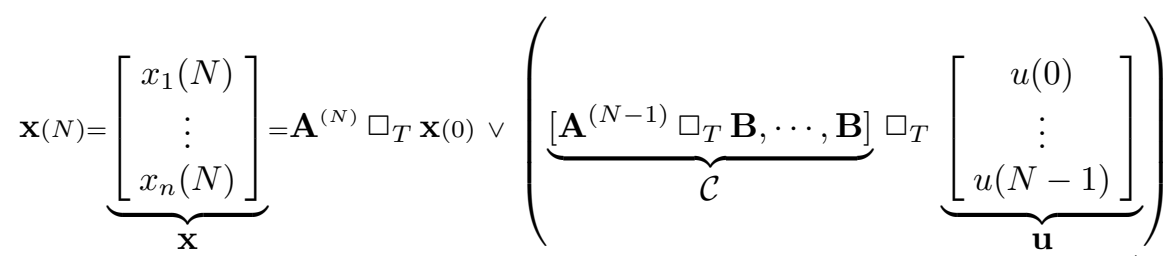

Assuming that the input is dominating the initial conditions, i.e., the second term $\mathcal{C} \square_{T} \mathbf{u}$ is not smaller than the first term $\mathbf{A}^{(N)} \square_{T} \mathbf{x}(0)$ of the right hand side, which is true if $\mathbf{x}(0)=\mathbf{0}$, we can rewrite the above as

$$
\mathcal{C} \square_{T} \mathbf{u}=\mathbf{x}
$$

Equations of the form (22) have been studied in $[10,11]$ in the context of fuzzy relations. The next lemma provides a sufficient condition for their solvability. Let $\mathbb{N}_{n}=\{1,2, \ldots, n\}$.

Lemma 1 ([10,11]). Equation (22) has a solution if for any $i \in \mathbb{N}_{n}$ there exists $j \in \mathbb{N}_{n}$ such that $\{\mathcal{C}\}_{i j}=1$ and $\{\mathcal{C}\}_{k j}=0 \forall k \neq i$.

In general, the set of solutions of (22) forms a sup-semilattice. The greatest solution is given by

$$
\mathbf{u}=\mathcal{C}^{t} \square_{\xi} \mathbf{x}
$$

where $\xi$ is the adjoint scalar erosion of the dilation $T$ defined in (10). In certain applications the conditions of Lemma 1 can be restrictive. An important aspect in such cases is finding the reachable set $\mathcal{R}$, i.e., the set of state vectors $\mathbf{x}$ for 
which (22) is solvable. Let us first recall some definitions given in [11]. With the aid of $\xi$ and a related binary operation

$$
\zeta(a, b) \triangleq\left\{\begin{array}{cc}
0, & a<b \\
\inf \{x: T(a, x)=b\}, & a \geq b
\end{array}\right.
$$

we define the following three solution matrices:

$$
\{\hat{\Gamma}\}_{i j} \triangleq \xi\left(\left\{\mathcal{C}^{t}\right\}_{i j}, x_{j}\right),\{\bar{\Gamma}\}_{i j} \triangleq \zeta\left(\left\{\mathcal{C}^{t}\right\}_{i j}, x_{j}\right),\{\check{\Gamma}\}_{i j} \triangleq \zeta_{\min }\left(\bigwedge_{k}\{\hat{\Gamma}\}_{i k},\{\bar{\Gamma}\}_{i j}\right)
$$

where $\zeta_{\min }$ corresponds to the case where $T$ is the min norm. Given the above matrices, the greatest solution is the row-wise infimum of $\hat{\Gamma}$, the mean solution is the row-wise supremum of $\check{\Gamma}$, and the minimal solutions also result from $\check{\Gamma}$. Note that the greatest solution that results from the solution matrix $\hat{\Gamma}$ is identical to the one provided by the adjoint vector erosion in (23).

The reachable set $\mathcal{R}$ can be found via the following Lemma:

Lemma 2 ([10,11]). The state vector $\mathbf{x}$ belongs to the reachable set $\mathcal{R}$ iff for any $i \in N_{n}$ such that $x_{i} \neq 0$ the ith column of $\check{\Gamma}$ is not equal to $\mathbf{0}$.

Hence, if a desired state $\mathbf{x}$ belongs to $\mathcal{R}$, the control $\mathbf{u}$ given by (23) drives the system to this state $\mathbf{x}$.

If $\mathbf{x}$ does not belong to $\mathcal{R}$, then it may be sufficient to solve an approximate controllability problem that has some optimality aspects. Specifically, consider the problem of finding an optimal input vector $\mathbf{u}$ as solution to the following optimization problem:

$$
\text { Minimize }\left\|\mathbf{x}-\mathcal{C} \square_{T} \mathbf{u}\right\| \text { subject to } \mathcal{C} \square_{T} \mathbf{u} \leq \mathbf{x}
$$

where the norm $\|\cdot\|$ is either the $\ell_{\infty}$ or the $\ell_{1}$ norm. The optimal controllability solution is actually a lattice erosion $\mathbf{u}=\varepsilon(\mathbf{x})=\mathcal{C}^{t} \square_{\xi} \mathbf{x}$ identical to the greatest solution (23). $\varepsilon$ is the adjoint erosion of the dilation $\delta(\mathbf{y})=\mathcal{C} \square_{T} \mathbf{y}$. Its optimality can be proven simply by noting that $(\varepsilon, \delta)$ forms a lattice adjunction, and hence $\delta \varepsilon$ is an opening operator. Opening is always anti-extensive, and hence $\delta(\varepsilon(\mathbf{x})) \leq \mathbf{x}$. Therefore, $\mathbf{u}=\varepsilon(\mathbf{x})$ is the largest solution with $\delta(\mathbf{u}) \leq \mathbf{x}$.

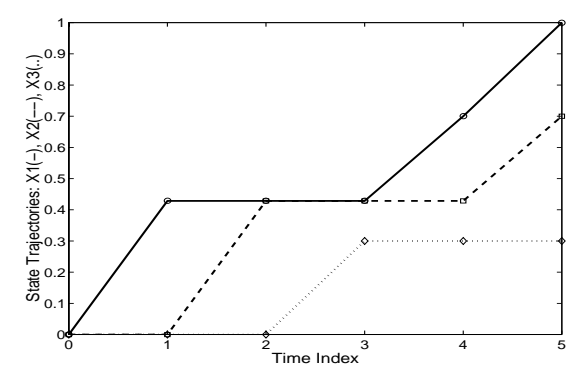

Fig. 1. State trajectories from initial state at $k=0$ to a desired state at $k=5$. 
Example: Consider a max- $T$ control system with $T(a, b)=a b, n=3$ states, $p=1$ input, and

$$
\mathbf{A}=\left[\begin{array}{ccc}
0.2 & 0.8 & 0.1 \\
1 & 0.3 & 0.2 \\
0 & 0.7 & 1
\end{array}\right], \quad \mathbf{B}=\left[\begin{array}{l}
1 \\
0 \\
0
\end{array}\right], \quad \mathbf{x}(0)=\left[\begin{array}{l}
0 \\
0 \\
0
\end{array}\right], \quad \mathbf{x}(N)=\left[\begin{array}{c}
1 \\
0.7 \\
0.3
\end{array}\right]
$$

This system can drive the initial state $\mathbf{x}(0)$ to the desired $\mathbf{x}(N)$ in $N=5$ steps by using the following scalar control signal

$$
[u(0), u(1), u(2), u(3), u(4)]^{t}=[0.4286,0.4286,0.4286,0.7,1.0]^{t}
$$

Figure 1 shows the state trajectories.

The above ideas on the controllability problem can also be applied to the observability problem. A max-T control system is observable if we can estimate the initial state by observing a sequence of output values. This can be done if the following system of nonlinear equations can be solved:

$$
\left[\begin{array}{c}
y(0) \\
\vdots \\
y(n-1)
\end{array}\right]=\underbrace{\left[\begin{array}{c}
\mathbf{C} \\
\vdots \\
\mathbf{C} \square_{T} \mathbf{A}^{(n-1)}
\end{array}\right]}_{\mathcal{O}} \square_{T} \mathbf{x}(0) \vee[h(n-1), \cdots, h(0)] \square_{T}\left[\begin{array}{c}
u(0) \\
\vdots \\
u(n-1)
\end{array}\right]
$$

This max- $T$ matrix equation can be solved either exactly or approximately by using the same methods as for the controllability equation.

\section{References}

1. G. Cohen, P. Moller, J.P. Quadrat and M. Viot, "Algebraic Tools for the Performance Evaluation of Discrete Event Systems", Proc. IEEE, vol. 77, pp. 39-58, Jan. 1989.

2. R. Cuninghame-Green, Minimax Algebra, Springer-Verlag, New York, 1979.

3. H.J.A.M. Heijmans, Morphological Image Operators, Acad. Press, 1994.

4. E. W. Kamen, "An Equation-based Approach to the Control of Discrete Event Systems with Applications to Manufacturing", in Proc. Int'l Conf. on Control Theory \& its Applications, Israel, Oct. 1993.

5. G. J. Klir and B. Yuan, Fuzzy Sets and Fuzzy Logic: Theory and Applications, PrenticeHall, 1995.

6. P. Maragos, "Lattice Control: General Theory", manuscript in preparation.

7. P. Maragos and S. Tzafestas, "A Lattice Calculus Unification of Min-Max Control Systems of the Morphological and Fuzzy Type", Proc. Int'l Symp. on Soft Computing in Engineering Applications (SOFTCOM-98), Athens, pp. 247-248, June 1998.

8. P. Maragos and S. Tzafestas, "Min-Max Control Systems with Application to Discrete Event Dynamic Systems", in Advances in Manufacturing: Decision, Control, and Information Technology, (S.G. Tzafestas, Ed.,) Springer-Verlag, London, 1999, pp. 217-230.

9. J. Serra, Ed., Image Analysis and Mathematical Morphology, Vol.2: Theoretical Advances, NY: Acad. Press, 1988.

10. G. Stamou and S. Tzafestas, "Fuzzy Relation Equations and Fuzzy Inference Systems: An Inside Approach", IEEE Trans. Syst. Man \& Cybernetics: Part B, Dec. 1999.

11. G. Stamou and S. Tzafestas, "Resolution of Composite Fuzzy Relation Equations Based on Archimedean Triangular Norms", Fuzzy Sets and Systems, to appear.

12. L. A. Zadeh, "Fuzzy Sets", Information and Control, vol. 8(3), pp. 338-353, 1965. 